\title{
Relaciones entre aprendizaje inicial de la lengua escrita, métodos de enseñanza y estilo cognitivo'
}

\author{
Relationships among initial learning \\ of written language, teaching methods and cognitive style
}

\author{
Lida Rincón C.* \\ Christian Hederich M.**
}

\section{Resumen}

Esta investigación examina el efecto diferencial de dos metodologías distintas para la enseñanza de la escritura (método silábico y método global) en el proceso de aprendizaje de niños con estilo cognitivo diferente, según la dimensión de dependencia-independencia de campo. La investigación se desarrolló en una institución educativa pública con niños entre los cinco y siete años de edad, que se encontraban cursando preescolar y primer grado. Gracias a los datos obtenidos se encontró, primero, que el método silábico tiende a favorecer el proceso de aprendizaje de los sujetos con tendencia a la independencia de campo y, segundo, que el método global tiende a ser más equitativo que el silábico, en tanto parece no favorecer más a un estilo cognitivo sobre otro.

\section{Palabras clave}

Aprendizaje, escritura, métodos de enseñanza, estilo cognitivo.

\section{Abstract}

This research examines the differential effects of two distinct methodologies for writing teaching (syllabic method and global method) in children learning process, according with the cognitive style, in field dependence-independence dimension. The research took place at a public school with children between five and seven years old, students from kinder and first grade. Thanks to the obtained data we find, firstly, that syllabic method tends to favor the learning process in subjects who tend to be field independents and, secondly, that global method tends to be more equitable than syllabic, because it doesn't seeming to favor any cognitive style.

Keywords

Learning, writing, teaching methods, cognitive style.

Artículo recibido el 11 de septiembre de 2008 y aprobado el 14 de noviembre de 2008.

* Ms. profesora Universidad Pedagógica Nacional e investigadora grupo Gipelec (Colciencias). Correo electrónico: ljrincon@pedagogica.edu.co

* Ph. D. profesor titular Universidad Pedagógica Nacional, Bogotá, Colombia. Investigador del Grupo de Estilos Cognitivos. Director de la tesis.

Correo electrónico: hederich@pedagogica.edu.co

1 El presente artículo expone algunos de los resultados de una tesis para el grado de Magister en Educación de la Universidad Pedagógica Nacional. 


\section{Introducción}

El propósito de este artículo es examinar el efecto diferencial de la aplicación de dos enfoques metodológicos distintos para la enseñanza de la escritura (método silábico y método global) sobre su aprendizaje en niños con estilos cognitivos diferentes, en la dimensión de dependencia-independencia de campo. Para este fin, el estudio realizado siguió un diseño de carácter analítico, descriptivo comparativo y pre-experimental pre-postest con dos grupos no equiparables.

Implícito en el propósito de la investigación está el pensamiento de que la actividad de la escritura comporta un fuerte componente cognitivo para su aprendizaje y que en él es posible identificar tendencias de funcionamiento expresables en la forma de estilos cognitivos diferentes. Por tal motivo, es fundamental identificar el modo en que tal componente ha sido abordado por las ciencias cognitivas. En esta medida, los aportes que la psicología cognitiva ha hecho al respecto confirman que la escritura, más que tratarse de un producto, se trata de un proceso de resolución de problemas que implica conductas cognitivas como la planificación, la organización, la estructuración y la revisión (Hull, 1996: 175). Esto lleva a pensar que, en tanto proceso cognitivo, la escritura requiere no sólo del dominio de funciones mentales elementales, sino de funciones superiores que implican el diseño de estrategias, la formulación de metas u objetivos, la recuperación de información en la memoria, la elaboración y la reflexión, entre otros procesos.

Otro de los aspectos desarrollados en el estudio de la escritura es el de la caracterización de la psicogénesis de la escritura. Al respecto, autores como Ferreiro (1983), Tolchinsky (1993) y Benveniste (2002) presentan estudios que buscan comprender la construcción cognitiva que conduce a los niños a dominar el código escrito. Ferreiro (1983) explica la existencia de tres niveles de construcción de hipótesis por los que los niños se conducen hasta acceder completamente al código convencional de la escritura; el primer nivel es el de distinción entre dibujo-grafía, el segundo es el de control de cantidad y calidad y, finalmente, el nivel de relación entre sonido y grafía.

En el plano de la enseñanza, han surgido dos grandes enfoques que encierran diferentes métodos para abordar el aprendizaje de la lengua escrita en los primeros años, interesados, cada uno, en promover el desarrollo de habilidades cognitivas que se entienden como importantes. Tales enfoques son: el sintético y el analítico o global. Para el caso de este estudio se trabajó específicamente con el método silábico (enfoque sintético) y el método global (enfoque analítico). Por un lado, los métodos que forman parte de un enfoque sintético se centran en el dominio del nivel fonológico como pauta inicial para el aprendizaje de la escritura (Cuetos, 1991: 107). En esta medida, una de las habilidades prioritarias por desarrollar es el conocimiento de la correspondencia fonema-grafía que, una vez dominada, conduce a la comprensión de las palabras. Desde esta perspectiva se privilegia el acceso a la información lingüística mediante la ruta fonológica. Por otro lado, los métodos del enfoque analítico se concentran en el principio comunicativo de la escritura, lo que conduce a centrar la atención en los procesos de producción de textos, incluso si los niños aún no dominan la correspondencia fonemagrafía. Así, desde este punto de vista, se privilegia el acceso a la información lingüística desde la "ruta visual”. Este enfoque está más relacionado con la teoría de la psicogénesis de la escritura descrita por Ferreiro (1983).

Por su parte, el término estilo cognitivo, tal y como se trabaja en esta investigación, se define como una tendencia o modo individual, estable y consistente de procesar la información (Hederich, 2007: 24-25). Los estudios en torno a este tópico han descrito numerosas dimensiones (Kagan et al., 1964; Klein, 1954; Reinert, 1976; citados por Abraham, 1983: 18). No obstante, el presente trabajo se centra únicamente en la dimensión dependencia-independencia de campo, DIC, definida por Witkin en los años cincuenta. Esta dimensión de estilo cognitivo alude a la manera en que un individuo percibe las partes separadas de una imagen, problema o situa- 
ción compleja, frente a la tendencia de procesar la información de forma global, teniendo en cuenta la imagen, problema o situación en su forma completa (Witkin et al., 1971). En cuanto a la primera tendencia mencionada, mediante ésta se define un sujeto independiente de campo, mientras que la segunda define a uno dependiente o, siguiendo algunas propuestas de redefinición, un sujeto "sensible al medio".

De este modo, siendo el estilo cognitivo un constructo psicológico que permite diferenciar a los individuos respecto de su tendencia al privilegiar ciertas operaciones cognitivas, éste puede considerarse como un factor que incide en el logro de aprendizaje de la escritura, dependiendo del método que se use. Es así como se hace posible encontrar alguna diferencia en lo que respecta al aprendizaje de la escritura, según las tendencias estilísticas de los individuos, es decir, de acuerdo con la manera en que procesan la información lingüística. En esta medida, es fundamental mirar con detenimiento la relación que podría existir entre las operaciones cognitivas que cada método promueve y las preferencias cognitivas de cada tendencia estilística en la dimensión dependencia-independencia de campo.

En concordancia con esto, se plantean dos hipótesis por verificar: En primer lugar, es posible decir que al trazarse ejercicios en los cuales los pares puedan comparar, confrontar y validar o invalidar las hipótesis entre sí, además de proponer ejercicios de escritura colectiva, el método global podría favorecer en mayor medida al sujeto sensible al medio que al independiente; en tanto el primero es más tendente a recibir la influencia de factores ambientales como la interacción con otros.

Por otro lado, el carácter sintético (va de las partes al todo) del método silábico posiblemente favorecería el aprendizaje de la escritura en sujetos con una tendencia a la independencia de campo. Este método exige de parte del sujeto, primeramente, el reconocimiento de la unidad mínima de la lengua escrita y luego la reestructuración de este conocimiento para combinar letras, conformar palabras, construir oraciones, entre otros. Este tipo de métodos plantean un ejercicio individual de aprendizaje, no se requiere de mayor interacción con otros. Desde esta perspectiva, y entendiendo que el sujeto independiente se caracteriza por ser más analítico y con una amplia capacidad de reestructuración perceptual, es posible lanzar esta segunda hipótesis.

\section{Antecedentes}

La revisión bibliográfica sobre los efectos diferenciales, por estilo cognitivo, de la aplicación de métodos distintos sobre el aprendizaje de la escritura no permitió establecer antecedentes directos al presente proyecto, en la medida en que no se hallaron aproximaciones empíricas previas que relacionaran el estilo cognitivo con los métodos de aprendizaje inicial de la escritura. Sin embargo, considerando la escritura como una actividad que requiere instrucción deliberada para su desarrollo y que no se adquiere de la misma manera que la lengua hablada, puede llegar a establecerse una relación entre ésta y el aprendizaje de una segunda lengua. $\mathrm{Al}$ respecto, se encontraron estudios sobre procesos de aprendizaje de una segunda lengua que han intentado descubrir la manera en que la correlación entre los estilos cognitivos y los métodos de enseñanza resulta ser predictora del logro de aprendizaje en los sujetos. Autores como Abraham (1983), Bialystok (1992), Fuller (1988) y Johnson \& Rosano (1993) han investigado, por una parte, acerca de la relación entre los estilos cognitivos y los procesos metalingüísticos $y$, por otra parte, acerca de la incidencia de estos en el aprendizaje de una lengua extranjera. En general, estos trabajos han demostrado que es posible explicar en cierta medida la manera en que los sujetos perciben y procesan información de tipo lingüístico por medio de los efectos diferenciales del estilo cognitivo.

Fuller (1988) quiso averiguar si existía una relación entre el estilo cognitivo en la dimensión de dependencia-independencia de campo, DIC, y la ejecución de la lengua objeto, según la orientación metodológica y si la percepción de los estudiantes sobre el aprendizaje de la lengua extranjera se relacionaba con dicha orientación y con el propio estilo cognitivo en la dimensión dic. Luego de la aplicación de pruebas relacionadas con el aprendizaje de la 
segunda lengua y con la determinación del estilo cognitivo de los estudiantes² (seleccionadas para cada uno de los cursos observados) no se encontró ninguna interacción entre el estilo cognitivo y la orientación metodológica; es decir, no se demostró que la orientación causara efectos en quienes tendían a la independencia, los que tendían a la sensibilidad o quienes se consideraron "intermedios".

Sin embargo, al observar de forma separada la DIC, se encontró que tanto los independientes como los intermedios tuvieron mayor puntaje que los sensibles en la prueba final de la orientación hacia el logro formal; pero, además, los independientes demostraron un nivel superior a los sensibles e intermedios en la prueba Oral Proficiency Interview, opI, empleada para la orientación en competencia comunicativa. En suma, Fuller (1988) demostró que los sujetos independientes del medio tenían ventajas en su actuación tanto en logros de tipo formal de lenguaje, como en los de tipo funcional, de modo que la metodología para la enseñanza de una lengua extranjera no parecía causar algún efecto en estos sujetos.

Según los aportes de Fuller (1988) y con relación al aprendizaje de la lengua escrita, podría establecerse, por un lado, que la aplicación del método silábico se propone lograr un dominio de los aspectos formales de la lengua escrita más que desarrollar la competencia comunicativa, ya que hace énfasis en el aprendizaje de cada grafema y fonema como unidades mínimas del código escrito. Por otro lado, se entendería que la aplicación del método global busca desarrollar la competencia comunicativa, en tanto parte de los intereses y necesidades del niño, además, de privilegiar la posibilidad de producir textos y explorar la escritura, más que memorizar grafías. De acuerdo con esto, es posible plantearse una hipótesis acerca del comportamiento (en términos de aprendizaje) de los independientes de campo;

2 Las pruebas de lenguaje se seleccionaron según el énfasis de la metodología; así, para la medida del logro lingüístico se tomaron en cuenta los exámenes finales del curso y para la medida de la competencia comunicativa se aplicaron exámenes como el ACTFL/ ETS Oral Proficiency Interview, OPI, (Liskin-Gasparro, 1982). Para la medición del estilo cognitivo se aplico el eft (Witkin et al., 1971). entonces, podría decirse que el independiente tendría mejores resultados en ambos métodos; por el contrario, podría pensarse que los sensibles se verían beneficiados por los métodos globales, más que por los sintéticos. Lo que realmente importa al establecer una relación entre el planteamiento de Fuller (1988) y el propósito de este artículo es observar si realmente existe una incidencia de las metodologías en el proceso de aprendizaje y los resultados obtenidos tanto por los sensibles al medio como por los independientes y descubrir si es posible explicar la efectividad de una u otra metodología a partir de la dimensión DIC.

Por su parte, Bialystok (1992) encontró que la metodología de enseñanza o el tipo de programa no influye de manera determinante en la competencia de los sujetos en el aprendizaje de una lengua extranjera, sino más bien la importancia que le asignan al significado lingüístico, más que a la gramática. Bialystok (1992: 664) plantea que tanto los problemas metalingüísticos como los problemas de DIC requieren que la atención sea enfocada en un aspecto de la entrada (input), normalmente con la presencia de información sobresaliente desde un aspecto irrelevante. Teniendo en cuenta que el mecanismo encargado de la solución de problemas metalingüísticos es el control del proceso de atención, la autora sugiere que dicho proceso puede ser un mecanismo de dominio general, que de igual forma está implicado en la resolución de problemas que no corresponden al campo lingüístico; en este caso, se refiere a las tareas de identificación de DIC. En otras palabras, el control de la atención se requiere tanto en las tareas de reestructuración perceptual como en las de carácter metalingüístico, lo que explica que los sujetos independientes de campo sean exitosos en ambas.

Desde los aportes de Bialystok (1992), para caracterizar el proceso de aprendizaje de la lengua escrita desde dos programas metodológicos distintos, es importante revisar el comportamiento de los sujetos según su estilo cognitivo. Existen dos posibilidades a observar. En primer lugar, se puede pensar que los sujetos independientes al medio se ven favorecidos por un método que privilegie el 
estudio formal de la lengua, es decir, el conocido con el nombre de método silábico, en tanto éste se inscribe en un enfoque analítico. En segundo lugar, se piensa en la incidencia que pueda tener el método global en los sujetos según su estilo cognitivo y es viable señalar que los sujetos sensibles tengan un comportamiento más favorable en este programa que en el otro, en tanto privilegia el desarrollo de las competencias comunicativas.

\section{Objetivos}

El objetivo principal del estudio propuesto en este artículo fue determinar el efecto diferencial de la aplicación de dos enfoques metodológicos distintos para la enseñanza de la escritura (silábico y global) sobre su aprendizaje en niños con estilos cognitivos diferentes en la dimensión DIC. Implícito en este objetivo, como se ha mencionado previamente, está el pensamiento de que la actividad de la escritura comporta un fuerte componente cognitivo para su aprendizaje y que en él es posible identificar tendencias de funcionamiento expresables en la forma de estilos cognitivos diferentes.

\section{Metodología}

\section{Muestra}

El estudio se desarrolló en una población de 33 estudiantes (18 niñas y 15 niños) de una institución educativa distrital. Los sujetos estaban distribuidos entre grado preescolar (19 niños, con una media de la edad de 5,98 ) y grado primero (14 niños, con una media de la edad de 6,36).

\section{Diseño}

La investigación siguió un diseño de tipo analítico, descriptivo y comparativo que diera cuenta del proceso de aprendizaje de la escritura en dos circunstancias diferentes, caracterizadas por dos métodos de enseñanza (silábico y global). Además, se buscó explorar el efecto de la variable de estilo cognitivo en la dimensión dependencia-independencia de campo sobre el logro de aprendizaje en las dos circunstancias anotadas. Por el tipo de diseño, puede decirse que fue una investigación pre-experimental pre-postest.

\section{Variables}

Dependiente: El nivel de logro de aprendizaje de la lengua escrita. Sus indicadores se expresan en términos de las dimensiones que se reflejan en el ejercicio de escritura: Dimensión formal, semántica, léxico-gramatical y pragmática.

Independiente: El método de enseñanza, con los siguientes valores: método global y método silábico.

Asociada: El estilo cognitivo. En la dimensión de dependencia-independencia de campo, DIC, indicada por los resultados frente a la prueba CEFT.

\section{Instrumentos}

Para la recolección de los datos se emplearon tres tipos de instrumentos.

- El ceft, Children Embedded Figures Test, para establecer la tendencia estilística de los sujetos en la dimensión de dependencia-independencia de campo. Esta prueba consiste en la medición de la capacidad de reestructuración perceptual en la que se le presenta al sujeto una figura como modelo, la cual debe encontrar en varias tarjetas con imágenes compuestas.

- Prueba de situación de selección de notaciones diseñada por Tolchinsky (1993). Esta prueba está diseñada para determinar el nivel en el que se encuentran los niños al iniciar el año escolar, en cuanto a la diferenciación del sistema notacional lingüístico convencional, de los dibujos y otros símbolos. La prueba se divide en cuatro partes, según el tipo de ítems que se presentan: Elementos de notación escrita, elementos de notación numérica, combinaciones de notación escrita y combinaciones de notación numérica.

- Finalmente, para el postest, se diseñó un instrumento de producción de textos, en el que los niños debían crear el final de una narración que era contada por la docente. Para la evaluación del postest, se diseñó una rejilla que medía la pro- 
ducción en las dimensiones formal, semántica, léxico-gramatical y pragmática.

\section{Procedimiento}

Como parte del trabajo de campo, se diseñó cada intervención pedagógica, de acuerdo con método a aplicar, durante siete meses. Para el grupo uno (preescolar) se diseñó la propuesta basada en el método global. Para el grupo dos (primero) se diseñó la propuesta según el método silábico.

En el procesamiento de los datos obtenidos en el trabajo de campo, se empleó el programa estadístico sPss. Se construyó una matriz de datos con todas las variables, con el fin de correr un análisis de correlación y nivel de asociación entre éstas. A partir de los datos arrojados por los análisis se buscó la verificación de la hipótesis. En el caso del estilo cognitivo se procesaron los resultados de la prueba CEFT y se distribuyeron por rangos, para agrupar a los sujetos en tres escalas y así obtener la medida de estilo cognitivo. Para cada grado se establecieron los rangos de manera diferente, teniendo en cuenta el puntaje mínimo y el máximo.

En el procesamiento se corrieron pruebas descriptivas y exploratorias para todas las variables, con el fin de obtener medidas de tendencia central. En segundo lugar, se corrió un análisis a partir de tablas de contingencia, útiles para trabajar con variables categóricas, como lo son en este caso el pretest, el postest y el estilo cognitivo en la dimensión DIC. Del mismo modo, se emplearon estadísticos como el coeficiente de correlaciones Spearman para establecer la medida de asociación entre las variables pretest y postest y estilo cognitivo en cada uno de los grupos que conformaban la muestra.

\section{Resultados}

Para los resultados, se debió hacer un procesamiento por separado de cada grupo, esto debido a que los dos grupos no son estrictamente comparables entre sí, ya que los grados de escolaridad y, por tanto, las edades, son diferentes. El primer paso fue establecer una descripción estadística con respecto al estado inicial de la muestra (resultados del pretest) y el estado final (resultados del postest). El segundo análisis fue una correlación entre el pretest-estilo cognitivo y postest-estilo cognitivo, respectivamente, mediante el coeficiente de Spearman. A continuación, se presentan los resultados de primero, ya que se tomó como punto de referencia, en tanto este grupo ya había tenido la formación de preescolar. Los resultados iniciales obtenidos en primero serán útiles para evaluar el logro alcanzado por el grupo de preescolar.

\section{Resultados del grado primero}

En el pretest aplicado a primero (prueba de notaciones) se encontró que, en los elementos de notación escrita, un sujeto se ubicó en el nivel inicial y el resto del grupo se distribuyó entre el nivel intermedio y el avanzado, con siete y seis sujetos, respectivamente. Del mismo modo, en las combinaciones de notación escrita, se encuentran cuatro sujetos en el nivel inicial, nueve en el intermedio y uno en el avanzado. Al comparar estas frecuencias con las obtenidas para los casos de notación numérica se marca una diferencia llamativa: ningún sujeto se encontró en el nivel inicial; por el contrario, la mayoría alcanzó un nivel avanzado.

Gráfica 1: Niveles de logro en el prestest (primer grado método silábico).

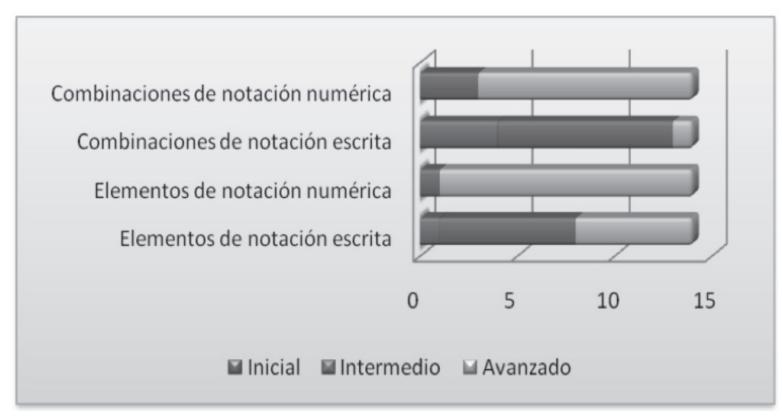

Según Tolchinsky (1993: 237-256), hay varios aspectos por tener en cuenta al interpretar los resultados de la prueba. En primer lugar, las combinaciones posibles en la notación numérica no tienen restricciones, pues todas las combinaciones son posibles y tienen significado (4566 ó 6645 ó $5466 . .$. ), aspecto que cambia en el caso de la notación escrita, en la que las posibilidades de combinación son limitadas y no todas poseen significado (scao ó csao no 
tienen significado, pero caso ó saco sí). Esto facilita el reconocimiento de las combinaciones numéricas como correctas en niños de primero, ya que para estos todas tienen sentido y son posibles; incluso, algunos niños respondían que las cifras largas correspondían a números de teléfono. En segundo lugar, los íconos que aparecen tanto en elementos de notación escrita y numérica como en combinaciones de ambos son rechazados en su mayoría por todos los sujetos del grupo, es decir, que los niños poseen la habilidad de discriminar entre lo que pertenece a notaciones convencionales y lo que no, que para ellos son dibujos (como l,p, f). ).

Tabla 1: Correlaciones de Spearmann entre pretest y estilo cognitivo en el grado primero.

\begin{tabular}{lll}
\hline Dimensiones de pretest & Valor & $\mathrm{P}$ \\
\hline Elementos de notación escrita & 0,236 & 0,417 \\
\hline Elementos de notación numérica & 0,367 & 0,197 \\
\hline Combinaciones de notación escrita & 0,319 & 0,266 \\
\hline Combinaciones de notación numérica & 0,230 & 0,428 \\
\hline
\end{tabular}

La Tabla 1 refleja el grado de correlación entre el pretest y el estilo cognitivo mediante el coeficiente de Spearman y su nivel de significación. Al revisar los valores, no es posible establecer relación entre los niveles del pretest y el estilo cognitivo de los sujetos.

El postest se aplicó una vez concluida la intervención basada en el método silábico. A partir del análisis de las pruebas según las dimensiones de la lengua escrita: formal, semántica, léxica-gramatical y pragmática se clasificaron los sujetos en tres niveles, de acuerdo con el puntaje obtenido en los ítems de cada dimensión:

Nivel inicial: que hace referencia a un conocimiento de la relación entre sonido y grafía, pero con un uso básico de las reglas gramaticales, sintácticas, semánticas y pragmáticas, manifestando un alto número de errores en la producción.

Nivel intermedio: que hace referencia al uso moderado de las reglas gramaticales, sintácticas, semánticas y pragmáticas, aunque con la aparición de algunos errores en la producción.

Nivel avanzado: que hace referencia a un mayor dominio en el uso de las reglas gramaticales, sintácticas, semánticas y pragmáticas.
La distribución de los casos aparece explicada la Gráfica 2.

Gráfica 2: Niveles de logro en el postest (primer grado método silábico).

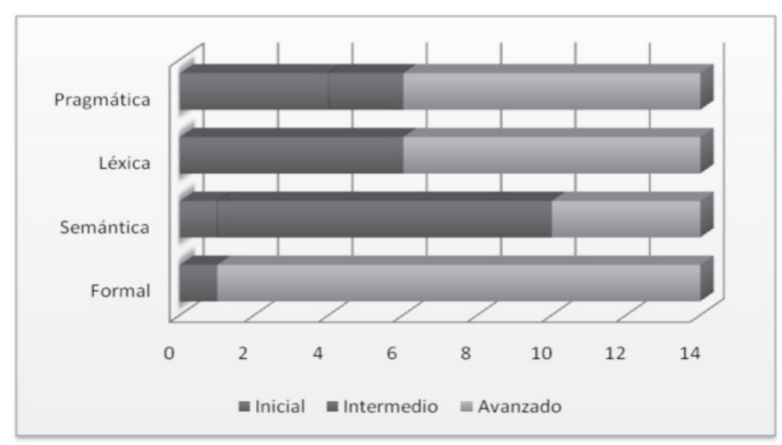

En general, al hacer una revisión de las cuatro dimensiones juntas, las estadísticas reflejan que el grupo de primero se halla distribuido, en su mayoría, en un nivel intermedio y un nivel avanzado, exceptuando un caso en el que el sujeto, a pesar de conocer las grafías, no las emplea en la construcción de palabras y textos comprensibles. Esto significa que los sujetos no sólo tienen conocimiento de la lengua escrita, sino que hacen un uso relativamente apropiado de ésta.

Tabla 2: Correlaciones entre postest y estilo cognitivo en el grado primero.

\begin{tabular}{lll}
\hline Dimensiones de postest & Valor & p. \\
\hline Dimensión formal & 0,367 & 0,197 \\
\hline Dimensión semántica & 0,680 & 0,008 \\
\hline Dimensión lexico - gramatical & 0,573 & 0,032 \\
\hline Dimensión pragmática & 0,553 & 0,040 \\
\hline
\end{tabular}

Los resultados generales arrojados por el coeficiente de Spearman, que relaciona postest con estilo cognitivo, reflejan datos muy interesantes en relación con la hipótesis planteada; es claro que los sujetos independientes al medio se vieron favorecidos en la aplicación del método silábico, en tanto sus resultados fueron notoriamente mejores que los intermedios y sensibles, específicamente en las dimensiones léxica ( $r h o=0,573 ; p=0,032)$, semántica (rho=0,680; $\mathrm{p}=0,008)$ y pragmática $(\mathrm{rho}=0,553$; $\mathrm{p}=0,04)$. Es importante resaltar este aspecto, en tanto forma parte de una de las afirmaciones esbozadas en la hipótesis de la presente investigación, la cual 
planteaba que los niños con tendencia a la independencia obtendrían mayores beneficios mediante el método silábico.

\section{Resultados de preescolar}

Para el caso de preescolar, los resultados del pretest reflejan un mayor dominio en el reconocimiento de elementos notacionales, más que en el de combinaciones. La Gráfica 3 presenta la distribución de los sujetos entre los niveles inicial, intermedio $\mathrm{y}$ avanzado.

Gráfica 3: Niveles de logro en el prestest (Preescolar método global).

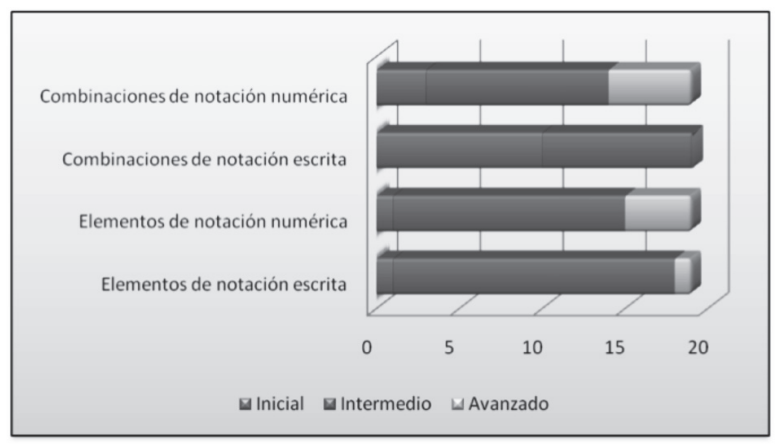

En cuanto a las combinaciones de notación, se marca una amplia diferencia. Como es evidente, los resultados más bajos de toda la prueba son los correspondientes a la condición de combinaciones de escritura, esto debido al poco conocimiento del código escrito por parte de los niños; pero no ocurre lo mismo con las combinaciones numéricas, esto lo explica Tolchinsky (1993: 253), reconociendo que, en el caso de las notaciones numéricas, todo tipo de combinaciones es posible y tiene significado, lo que no impone restricciones en la comprensión de las notaciones y en la asignación del rótulo de cifras.

Es de resaltar, como lo plantea Tolchinsky en las conclusiones de su investigación, que los sujetos desde los años de la infancia problematizan en torno a los principios de dominios notacionales; ellos conocen acerca de la escritura, aún antes de adquirir las herramientas necesarias para su uso con fines comunicativos (Tolchinsky, 1993: 255-256). En su mayoría, los niños establecen diferencias entre los distintos dominios y saben distinguir entre notaciones convencionales y las icónicas. Los resultados de los sujetos de preescolar comprueban las afirmaciones aquí establecidas, es decir, que sin haber cursado otros grados escolares son capaces, en alguna medida, de distinguir entre dominios y de reconocer elementos que les son útiles para la escritura y los que no lo son, aunque su nivel no sea el mismo que el obtenido por primer grado.

Tabla 3: Correlaciones de Spearmann entre pretest y estilo cognitivo en preescolar.

\begin{tabular}{lll}
\hline Dimensiones de pretest & Valor & $\mathrm{p}$ \\
\hline Elementos de notación escrita & $-0,221$ & 0,363 \\
\hline Elementos de notación numérica & $-0,447$ & 0,055 \\
\hline Combinaciones de notación escrita & 0,205 & 0,399 \\
\hline Combinaciones de notación numérica & $-0,137$ & 0,575 \\
\hline
\end{tabular}

Haciendo una mirada general de los resultados de la correlación entre pretest y estilo cognitivo, es posible observar, por medio de los valores expresados en forma negativa, que en las dimensiones relacionadas con las notaciones numéricas los sujetos independientes alcanzaron niveles inferiores a los obtenidos por los sensibles y los intermedios (según la DIC). Algo similar puede verse en la dimensión de elementos de notación escrita. Al parecer, el estado inicial de los sujetos de preescolar refleja una ligera desventaja inicial por parte de los individuos con tendencia a la independencia. No obstante, sólo el nivel de significación arrojado en el ítem de elementos de notación numérica $(\mathrm{p}=0,05)$ podría favorecer el apoyo de esta tesis.

El comportamiento de los sujetos por cada una de las dimensiones del postest se expresa en la Gráfica 4, presentando el número de casos encontrado en cada nivel. Esta información se obtuvo luego de hacer una evaluación prueba por prueba para clasificar a los sujetos según el nivel. En la dimensión formal, en términos cualitativos, los sujetos que alcanzaron un nivel avanzado son aquellos que emplean grafías convencionales, siguen las reglas de combinación posibles, fragmentan correctamente las palabras y escriben las letras de tal manera que son distinguibles o legibles. Es de resaltar que ningún caso se ubicó en el nivel inicial. 
En la dimensión semántica, la mayoría de sujetos no superaron el nivel inicial en lo que respecta a seguir una secuencia temática y a la construcción de oraciones con sujeto, es decir, no predicativas. Esto se debe a que hay sujetos que aún no han alcanzado el nivel alfabético, tal y como lo define Ferreiro (1983), por lo que emplean grafías convencionales, pero construyen palabras que no tienen un significado convencional o, en otros casos, escriben palabras reales, pero no producen el texto con las características que se solicitaron.

En la dimensión léxica, cuatro sujetos lograron alcanzar el nivel avanzado, lo que implica que usaron palabras verdaderas o reales y, además, emplearon artículos, conjunciones, preposiciones, de modo que asignaban mayor sentido a las frases. Los sujetos que alcanzaron un nivel intermedio en ocasiones cometían errores en la escritura de las palabras y obviaban el uso de artículos y conjunciones, sin embargo, sus textos eran comprensibles; en otras palabras, era posible decodificarlos y develar su sentido. Los ocho sujetos que permanecieron en el nivel inicial continúan escribiendo en grafías creadas por ellos mismos, sin emplear las convencionales.

En lo que respecta a la dimensión pragmática, los resultados indican que seis individuos alcanzaron un nivel avanzado y dos un nivel intermedio. Quienes avanzaron hasta el tercer nivel lograron responder a los requerimientos de la situación en términos de la creación de un texto narrativo, cumpliendo con las características generales de este género.

Gráfica 4: Niveles de logro en el postest (preescolar método global).

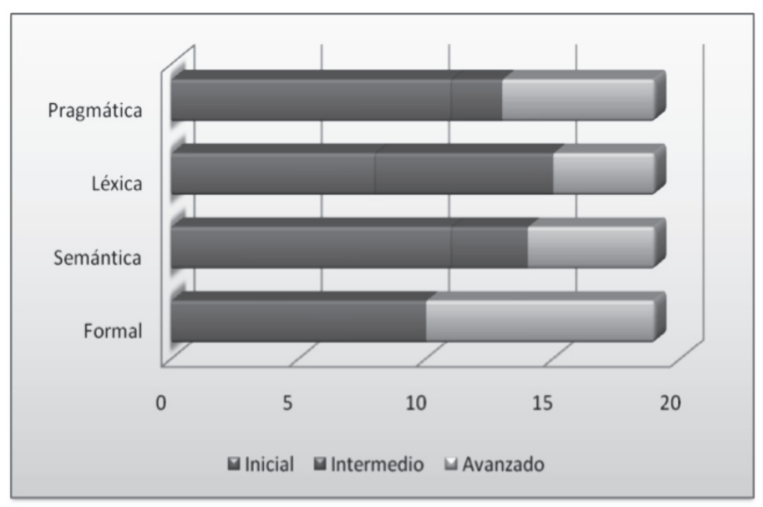

En el análisis de la correlación entre postest y estilo cognitivo (véase Tabla 4), el caso de preescolar difiere notoriamente del presentado en primero, en la medida en que en este grado los sujetos independientes tienden a ubicarse en niveles más bajos en comparación con los otros grupos de estilo cognitivo, lo cual es indicado por el hecho de que los valores del coeficiente de correlación son siempre negativos; aunque no es posible hablar de diferencias altamente significativas entre los grupos estilísticos, sí podría creerse que hay una muy leve correlación negativa, al menos en la dimensión formal y la semántica.

Estos resultados suscitan una reflexión en torno a la metodología de enseñanza aplicada para el grado de preescolar (método global). Si se revisa nuevamente la hipótesis planteada, no parece haber una confirmación de la afirmación que planteaba la posibilidad de que el método global privilegiaría mayormente a los sujetos sensibles al medio, en tanto está basado en el desarrollo de ejercicios de construcción textual colectiva, que requieren de comparar, confrontar y validar hipótesis. Sería mejor afirmar que es posible que el método global no incida más sobre una tendencia estilística que otra, pues los niveles de correlación no permiten establecer con certeza que la DIC explique claramente el logro de aprendizaje alcanzado por los sujetos que se instruyeron a partir del método global.

Tabla 4: Correlaciones entre postest y estilo cognitivo en el preescolar.

\begin{tabular}{lll}
\hline Dimensiones de postest & Valor & p. \\
\hline Dimensión formal & $-0,205$ & 0,399 \\
\hline Dimensión semántica & $-0,212$ & 0,383 \\
\hline Dimensión lexico - gramatical & $-0,077$ & 0,755 \\
\hline Dimensión pragmática & $-0,093$ & 0,705 \\
\hline
\end{tabular}

\section{Discusión}

Los resultados presentados dan a conocer los efectos diferenciales de la aplicación de los métodos silábico y global sobre el nivel de logro en escritura alcanzado por los niños con estilos cognitivos diferentes en la dimensión de dependencia-independencia de campo. Es importante recordar que los resultados de cada grupo se presentaron por separado debido 
a que los grupos no eran totalmente comparables por la diferencia de grado.

En primer lugar, fue indispensable reconocer el nivel en el que se encontraban los sujetos al inicio del proceso. El pretest (prueba de notaciones) permite clasificar a los sujetos de cada grupo en niveles de conocimiento del código escrito. Al respecto, es importante resaltar que tanto los niños de primero como los de preescolar poseían cierto conocimiento, aunque en diferente nivel, de las notaciones tanto numéricas como escritas. Según Tolchinsky (1993: 237), los niños, desde edades tempranas, incluso antes de acceder a la educación formal, ya poseen conocimiento acerca del código lingüístico y logran diferenciar entre lo que es un dibujo, una grafía del alfabeto y otro tipo de símbolos. Por supuesto, esto no implica que en estos niveles los niños necesariamente tengan un manejo pleno de la lengua escrita y que dicho manejo les permita usarla. Con respecto a esto, Benveniste (2002: 15) aclara que el concepto de "código" fue elaborado por los lingüistas para hacer la "notación" de las lenguas, de acuerdo con esto, se explica el principio consistente en que cada unidad sonora de la lengua tiene un signo gráfico correspondiente. Con base en esto, debe distinguirse entre el reconocimiento de notaciones y el ejercicio de escribir como tal. En este sentido, el reconocimiento de las notaciones (identificar los signos lingüísticos y distinguirlos de otros) significa una parte fundamental en ese proceso de adquisición de la lengua escrita, pero no asegura el hecho de que los niños escriban textos.

Al establecer la correlación entre el estado inicial de los niños, en cuanto al conocimiento del código escrito, y su tendencia estilística, se encontró, por una parte, que en el grado de preescolar había una ligera ventaja de los sujetos sensibles con relación a los otros, aunque el coeficiente rho no permite establecer correlaciones significativas entre la DIC y el pretest. Por otra parte, los resultados de primer grado reflejaron una leve ventaja de los niños con tendencia a la independencia, aunque en la condición de elementos de notación numérica los intermedios los hayan superado; esto se determinó a partir del estudio de número de casos. Los coefi- cientes de correlación arrojaron datos estadísticos que permiten establecer un grado de ventaja en los resultados del pretest en los sujetos independientes de campo.

A partir del reconocimiento del estado inicial de los sujetos, según su tendencia estilística, se buscaba determinar la manera en que la interacción entre las variables metodología de enseñanza y estilo cognitivo permite explicar el nivel de logro alcanzado al finalizar el proceso. Para esto, se hizo un análisis de correlación entre los resultados del postest y la variable de estilo cognitivo, lo que nos permite examinar nuevamente las hipótesis planteadas a partir de los resultados obtenidos:

En preescolar no se reflejan amplias diferencias estilísticas; apenas es evidente una ligera ventaja lograda por parte de los sensibles; no obstante, el coeficiente de correlación Spearman no permite establecer un nivel significativo como para afirmar que el método favorece más a unos que a otros según la DIC. Si se retoma la afirmación de la hipótesis, que planteaba que el método global probablemente beneficiaría más el proceso de aprendizaje de la escritura en los sujetos con tendencia a la sensibilidad, es necesario admitir que a partir de la contrastación con los resultados obtenidos no es posible validar del todo tal afirmación, aunque tampoco es pertinente negarla. Los datos obtenidos a partir del análisis conducen a pensar el método global como un método relativamente equitativo, es decir, que permite avanzar a los niños con diferencias estilísticas en el proceso de adquisición de la escritura de una forma ecuánime.

Además de lo expresado en la hipótesis, es esencial analizar el medio por el cual los sujetos expuestos al método global se relacionan con el código escrito. Es preciso recordar que el método global no segmenta en primer momento el lenguaje escrito para que los niños avancen en el reconocimiento de la correspondencia entre fonema y grafía, sino que se busca que mediante la interacción con su medio ambiente cercano asimilen la información de tipo verbal (escrita) y se les provee la posibilidad de experimentar con la escritura para que comprendan sus propiedades, su forma de construcción, sus carac- 
terísticas, etc. y que pongan a prueba sus hipótesis; expresado en otros términos, se busca respetar el proceso de "evolución de la alfabetización" que los niños experimentan aun antes de entrar a la educación formal (Ferreiro, 1983: 32-35).

Si se revisa este enfoque metodológico desde la discusión presentada por Bialystok (1992), es posible establecer una relación entre el método global y el análisis que la autora presenta con respecto a la resolución de tareas metalingüísticas. De este modo, al hablar de los dos componentes de la habilidad metalingüística (el control del proceso lingüístico y el análisis del conocimiento lingüístico), podría decirse que el método global demanda en un mayor grado el desarrollo del análisis del conocimiento lingüístico más que el control sobre el proceso, sin que esto indique que no se da lugar a este último. Esto significa que los sujetos son conducidos a hacer más explícito y más integrado con otro conocimiento el ejercicio de reorganización y de reestructuración de las representaciones del conocimiento lingüístico.

Bialystok (1992: 654) afirma que "el desarrollo de competencias lingüísticas en los niños parece conducirlos a través de lo conversacional, al conocimiento del código escrito y, finalmente, al dominio metalingüístico del uso de la lengua”. Este mismo proceso es el que se busca desarrollar desde la propuesta del método global, como se había mencionado, respetando el proceso de evolución de la alfabetización. Es así como se afirma que el énfasis del método se da sobre el componente de análisis del conocimiento, en el que los niños exploran la lengua a partir de sus propias representaciones y de la construcción y comprobación de hipótesis lingüísticas, todo esto a partir del reconocimiento del propósito comunicativo de la lengua. No obstante, la relativa ecuanimidad del método debe reafirmarse al entender que el componente de control del proceso tiene lugar, pero no es enfático. Bialystok (1992: 655) explica que este componente es fundamental para el desarrollo de tareas lingüísticas que tienen que ver con la parte formal del lenguaje y con la resolución de problemas en los que la solución requiere de atender aspectos de la información lingüística de entrada que no es sobresaliente o que no es usual; en el caso del aprendizaje de la escritura, se relacionaría con la comprensión de la información grafémica, lo que implica un conocimiento de la correspondencia fonema-grafía.

El control del proceso en este nivel es requerido en la medida en la que el niño debe esforzarse por atender a la información relevante, tratando de ignorar la irrelevante; en otras palabras, el niño necesita hacer un esfuerzo mayor para poder reconocer las grafías que forman parte de la escritura convencional y el sonido que le corresponde; mientras el niño no automatice este proceso, se demandará de un mayor control de la atención. El método global no ignora la relevancia de este conocimiento, pero no es el punto de partida para el proceso de aprendizaje de la lengua escrita; más bien se parte del reconocimiento de la psicogénesis de la escritura del niño y del propósito comunicativo de esta para conducirlo paulatinamente hacia el acceso completo al código convencional, lo que implica llegar al nivel de conciencia fonológica.

En lo que respecta al primer grado (método silábico), la discusión toma un curso diferente, en tanto los resultados demuestran que el método causó una notoria influencia sobre los sujetos con tendencia a la independencia. En el estado inicial, las correlaciones reflejan una leve relación entre el pretest y la DIC, lo que lleva a pensar que los sujetos independientes de este grupo ya se hallaban en una ligera ventaja. Sin embargo, tal y como lo demuestran los análisis de correlación entre el postest y la DIC, dicha ventaja permaneció y se enfatizó al terminar el proceso. Este resultado confirma la segunda afirmación de la hipótesis, en la que se aseveraba acerca de la influencia positiva que podría generar el método silábico en el proceso de aprendizaje de la escritura en los independientes.

Si se revisan los estudios de Bialystok (1992) y se aplican a la propuesta del método silábico, es evidente que se hace un énfasis mayor en el componente de control del proceso, en tanto le exige al niño desde el primer momento seleccionar información entre elementos relevantes e irrelevantes. Desde esta perspectiva, lo relevante es el dominio de la correspondencia fonema-grafía, y una vez logrado 
este conocimiento se pasa a otro proceso en el aprendizaje de la escritura, pero por un largo tiempo, el foco de atención está sobre este nivel, que incluye la "enseñanza sistemática de las reglas de conversión grafema-fonema [...] lo que significa que el niño tiene que ser capaz de segmentar las palabras en sus letras componentes y de asignar a cada letra el sonido que le corresponde" (Cuetos, 1996: 99).

Este método demanda del niño altos niveles de reestructuración de la información, requiere de segmentar el lenguaje en unidades mínimas y comprender la relación que se establece entre estas y los sonidos lingüísticos a los que representan. Es claro que este tipo de tareas lingüísticas requiere un alto control del proceso por parte de los niños que apenas acceden al código. Bialystok, mediante sus investigaciones, halló una correlación entre ciertas tareas metalingüísticas y lingüísticas y la independencia de campo, explicada a partir del componente de control del proceso que ambas requieren. En otros términos, algunas tareas metalingüísticas y la resolución de test como el CEFT demandan de un alto control atencional. Es claro que los sujetos independientes de campo tienen un mayor control de la atención, lo que los hace no sólo más efectivos en las tareas de reestructuración perceptual, sino en resolución de tareas metalingüísticas. Estos individuos automatizan los procesos de adquisición de la conciencia fonológica de manera más rápida, lo que les permite avanzar a niveles superiores. De acuerdo con estos hallazgos, se explica la relación encontrada entre el nivel de logro alcanzado por los independientes y la aplicación del método silábico.

Con base en los resultados obtenidos, no es posible concluir, por una parte, que el método global favorezca exclusivamente a los sujetos sensibles, lo que sí debe reconocerse es que es una propuesta que al parecer busca beneficiar a todos los sujetos, independientemente de su tendencia cognitiva. Por otra parte, puede concluirse que el método silábico sí orienta sus propuestas hacia la potenciación de las competencias de los sujetos independientes. En general, es evidente que sí existe una interacción entre los métodos de enseñanza de la lengua escrita y la dimensión de dependencia-independencia de campo a la hora de explicar el nivel de logro alcanzado por los sujetos; interacción que se manifiesta de forma diferente según el método. Del mismo modo, se hace evidente que los componentes de procesamiento lingüístico, descritos por Bialystok (1992), resultan determinantes en la influencia que los métodos ejercen sobre el aprendizaje de los niños con estilos cognitivos diferentes; esto debido a que el logro alcanzado por los sujetos dependerá de algún modo del énfasis que de estos se haga en el diseño. Además, se reafirma la conclusión de la autora acerca de la relación que establece el componente de control del proceso entre tareas metalingüísticas y la dimensión de independencia de campo.

Finalmente, hay un aspecto que es fundamental enunciar de nuevo y es el hecho de que los grupos de la muestra no son comparables entre sí, debido a la diferencia de grados, lo que implica desigualdad en términos de conocimiento de la lengua escrita. Esto deja abierta la invitación a futuros estudios para realizar un ejercicio comparativo desde grupos que conserven homogeneidad en aspectos como la edad y el nivel de escolarización, tomando una muestra más grande que la tomada para esta investigación, de modo que exista un mayor control de las variables. No obstante, no debe quitársele relevancia a los hallazgos del presente ejercicio investigativo, en tanto abre una línea de estudios, por una parte, relacionada con los efectos diferenciales de métodos de enseñanza en sujetos con estilos cognitivos diferentes y, por otra parte, relacionada con las implicaciones de los estilos cognitivos en la realización de tareas cognitivas involucradas en la escritura; aspectos que antes no habían sido abordados, además de aportar interesantes datos en cuanto a las relaciones entre las propuestas educativas y la teoría de los estilos cognitivos, que ha resultado tan fundamental en la comprensión de las diferencias individuales de los estudiantes. De modo que aspectos como la teoría de estilos cognitivos no pueden ser ignorados a la hora de reflexionar las prácticas educativas y proponer metodologías de enseñanza, considerando que los factores cognitivos como este tienen una incidencia evidente en los procesos de aprendizaje de los individuos. De esta manera, se 
ha logrado comprobar que en la medida en que los métodos educativos promueven ciertos procesos cognitivos, la DIC interfiere en la manera en que los

\section{Referencias bibliográficas}

Abraham, R. (1983). Relationships between use of the strategy of monitoring and the cognitive style. Studies in second language acquisition, 6, pp. 17-32.

Benveniste, C. (1998). Estudios lingüísticos sobre la relación entre la oralidad y escritura. Barcelona: Gedisa.

Benveniste, C. (2002). La escritura, irreductible a un "código". En E. Ferreiro (Ed.), Relaciones de (in)dependencia entre oralidad y escritura. Madrid: Gedisa

Bialystok, E. (1992). Attentional control in children's metalinguistic performance and measures of field independence. Developmental Psychology, 4, pp. 654-664.

Cuetos, F. (1991). La psicología de la escritura. Madrid: Editorial Escuela Española.

Cuetos, F. (1996). La psicología de la lectura. Barcelona: Editorial Praxis.

Ferreiro, E. (1983). Psicogénesis de la escritura. En C. Coll (Comp.). Psicología Genética y aprendizajes escolares. Madrid: Siglo XXI Editores.

Ferreiro, E. (2002). Relaciones de (in)dependencia entre oralidad y escritura. Barcelona: Gedisa.

Fuller, E. (1988). The relationship of field dependent/ independent cognitive style to Spanish language achievement and proficiency: a preliminary report. The Modern Language Journal, 72, pp. 21-30.

Grupo de investigación de audición y lenguaje, GIAL. (2008) Tema 6. La lectoescritura. España: Universidad de Extremadura. Consultado el 20 de abril de 2008 en: http://www.unex.es/gial/docencia/asignaturas/dhl/documentos\%20b\%E1sicos/Lecciones\%20 Hipertextualizadas/TEMA\%206.htm

Hayes J. (1996). Un nuevo marco para la comprensión de lo cognitivo y lo emocional en la escritura. The Science of Writing, 1, p. 72.

Hederich, C. (2007). Estilos cognitivos en la dimensión de independencia-dependencia de campo: influencias culturales e implicaciones para la educación. Tesis Doctoral de la Universidad Autónoma de Barcelona. Colección Tesis Doctorales, 4. Universidad Pedagógica Nacional. estudiantes asumen el proceso y alcanzan ciertos niveles de logro. 11

Hull, G. A. (1996). La investigación en escritura: La construcción de una comprensión cognitiva y social de la composición. En L. Resnick y L. Klopfer (Eds.), Currículum y cognición (pp. 134-155). Buenos Aires: Aique.

Jiménez, J., Rodrigo, M. y Hernández, I. (1999). Procesos de aprendizaje y desarrollo de la lectura. En De Vega y Cuetos (Coord). Psicolingüística del Español. Madrid: Trotta.

Jolibert, J. (1991). Formar niños productores de texto. Chile: Editorial Dolmen.

Johnson, J. y Rosano, T. (1993). Relation of cognitive style to metaphor interpretation and second language proficiency. Applied Psycholinguistics, 14, pp. 159-175.

Liskin-Gasparro. J. (1982). ETS Oral Proficiency Testing Manual. Princeton, Nueva Jersey: Educational Testing Service.

Ministerio de Educación Nacional. (1996). Lineamientos curriculares de lengua castellana. Bogotá: Editores.

Ramírez, M. y Castañeda, A. (1974). Cultural democracy, biocognitive development and education, Nueva York: Academic Press.

Tolchinsky, L. (1993). Aprendizaje del lenguaje escrito: procesos evolutivos e implicaciones didácticas. Barcelona: Universidad Pedagógica Nacional. Secretaría de Educación Pública.

Tolchinsky, L. (1995). ¿Aprender sonidos o escribir palabras? En A Teberosky y L. Tolchinsky (Comp.) Más allá de la alfabetización. Buenos Aires: Santillana.

Witkin, H. y Goodenough, D. (1981). Estilos cognitivos: naturaleza y orígenes. Madrid: Pirámide.

Witkin, H. Oltman, K., Raskin, E. y Karp, S. (1971). A manual for the Embedded Figure Test. Palo Alto, California: Consulting Psychologist Press.

Witkin, H., Oltman, K., Raskin, E. y Karp, S.A (1971). A manual for the Embedded Figure Test. Palo Alto, California: Consulting Psychologist Press. 